\title{
Transport phenomena in a plasma of confining gluons
}

\author{
Radoslaw Ryblewski ${ }^{1, a}$ \\ ${ }^{1}$ The H. Niewodniczański Institute of Nuclear Physics, Polish Academy of Sciences, \\ PL-31342 Kraków, Poland
}

\begin{abstract}
The plasma of confining gluons resulting from the Gribov quantization is considered. In the fluid dynamical framework the non-equilibrium properties of the system are studied. In the linear response approximation the formulas for the bulk, $\zeta$, and shear, $\eta$, viscosities of the plasma are calculated analytically. Surprisingly, the approximate scaling of the $\zeta / \eta$ ratio reveals the strong-coupling properties of the system under consideration.
\end{abstract}

\section{Introduction}

The experimental data measured in ultra-relativistic heavy-ion collisions at RHIC (Relativistic HeavyIon Collider) and the LHC (Large Hadron Collider) energies suggests that the new state of strongly interacting matter created in these reactions, called the quark-gluon plasma (QGP), to a good approximation behaves as a fluid with the smallest viscosity in Nature. This observation triggered enormous interest in the development of relativistic viscous [1-22], and, more recently, anisotropic [23-29] fluid dynamics.

The successful application of the fluid dynamical modelling requires, in addition to the initial conditions and the freeze-out prescription, some a priori knowledge about the thermodynamic as well as the transport properties of the underlying microscopic theory, namely quantum chromodynamics (QCD). While the lattice QCD simulations results on the equation of state at large and intermediate temperatures are already quite reliable [30,31], and agree well with the results of the resummed perturbation theory methods [32-34], there is still a lot of study required to understand the dynamic properties of the system [35]. In the case of charge-free medium the two most important transport coefficients which enter the theory already at the first order in the gradient expansion are the bulk, $\zeta$, and shear, $\eta$, viscosities.

In this work we review the main results of our recent investigations [36, 37] concerning the bulk and shear viscosities in the plasma made of confining gluons resulting from the Gribov-Zwanziger (GZ) quantization [38, 39]. We show that the GZ plasma has certain features which are characteristic for strongly-coupled systems.

\section{Gribov dispersion relation}

The Gribov quantization amounts to fixing the residual gauge transformations remaining after the Fadeev-Poppov procedure. In the Coulomb gauge it results in the modification of the dispersion

a e-mail: Radoslaw.Ryblewski@ifj.edu.pl 
relation for gluons in the infrared regime [38]

$$
E(\mathbf{k})=\sqrt{\mathbf{k}^{2}+\frac{\gamma_{\mathrm{G}}^{4}}{\mathbf{k}^{2}}} .
$$

In consequence the soft gluons are effectively removed from the physical spectrum, which leads to the confining properties of the system. At the same time a new scale, the so called Gribov scale $\gamma_{\mathrm{G}}$, is introduced which may be found by a self-consistent solution of the gap equation $[38,39]$ and recently it was shown to be directly related to the (chromo)magnetic scale [40, 41]. It was Zwanziger who generalized Gribov approach to the case of finite temperatures and presented its qualitative aggreement with pure glue lattice data [39]. Recently various interesting properties of the GZ approach have been demonstrated [41-48] which makes it an interesting playground for a study of the impact of confining properties of SU(3) Yang-Mills theory on the plasma dynamics.

\section{Fluid dynamical description of the Gribov-Zwanziger plasma}

For bulk and shear transport coefficients it is sufficient to consider the simplest $(0+1)$-dimensional longitudinally boost-invariant and transversally homogeneous system, which, as a matter of fact, resembles well the early-time dynamics of ultra-relativistic heavy-ion collisions. In this case the most general form of the energy-momentum tensor is

$$
T^{\mu v}=\left(\varepsilon+P_{T}\right) u^{\mu} u^{v}-P_{T} g^{\mu \nu}+\left(P_{L}-P_{T}\right) z^{\mu} z^{v},
$$

where the four-velocity is $u^{\mu}=(t, 0,0, z) / \tau$, with $\tau=\sqrt{t^{2}-z^{2}}$ being proper time, and $z^{\mu}=$ $(z, 0,0, t) / \tau$. In Eq. (2) the variables

$$
\begin{aligned}
\varepsilon & =\int \mathrm{d} K E(k \cdot u) f\left(\tau, w, k_{\perp}\right), \\
P_{L} & =\int \mathrm{d} K \frac{w^{2}}{\tau^{2} E(k \cdot u)}\left[1-\frac{\gamma_{\mathrm{G}}^{4}}{(k \cdot u)^{2}}\right] f\left(\tau, w, k_{\perp}\right), \\
P_{T} & =\int \mathrm{d} K \frac{k_{\perp}^{2}}{2 E(k \cdot u)}\left[1-\frac{\gamma_{\mathrm{G}}^{4}}{(k \cdot u)^{2}}\right] f\left(\tau, w, k_{\perp}\right),
\end{aligned}
$$

denote the energy density, transverse and longitudinal pressure, respectively [36, 37]. Here we introduced the phase space distribution function $f, \int \mathrm{d} K(\ldots)=\frac{g_{0}}{(2 \pi)^{3}} \int_{-\infty}^{\infty} \frac{\mathrm{d} w}{\tau} \int \mathrm{d}^{2} k_{\perp}(\ldots), k^{\mu}=(|\mathbf{k}|, \mathbf{k})$ and $w=k_{\|} t-k^{0} z$. In Eqs. (3)-(5) we employed the GZ dispersion relation (1) which has the following covariant form $[36,37]$

$$
E(k \cdot u)=\sqrt{(k \cdot u)^{2}+\frac{\gamma_{\mathrm{G}}^{4}}{(k \cdot u)^{2}}} .
$$

The dynamical properties of the system follow from the energy and momentum conservation, $\partial_{\mu} T^{\mu v}=0$, which reduces to the single differential equation

$$
\frac{\mathrm{d} \varepsilon}{\mathrm{d} \tau}+\frac{\varepsilon+P_{L}}{\tau}=0
$$

In Eq. (7) one can identify bulk and shear viscous pressure corrections through

$$
\pi=2\left(P_{T}-P_{L}\right) / 3
$$


and

$$
\Pi=P-P_{\mathrm{GZ}},
$$

where $P=\left(P_{L}+2 P_{T}\right) / 3$ and $P_{\mathrm{GZ}}$ is the pressure in the GZ equilibrium, that is, in the case where $f$ has the Bose-Einstein form $f_{\mathrm{GZ}}=\{\exp [E(k \cdot u) / T]-1\}^{-1}$ and the temperature $T$ follows from the Landau matching condition, $\varepsilon(T)=\varepsilon_{\mathrm{GZ}}\left(T, \gamma_{\mathrm{G}}\right)$. Once the system is in the vicinity of the local thermal equilibrium the pressure corrections are well described by the Navier-Stokes expressions

$$
\pi=\frac{4}{3} \frac{\eta}{\tau}, \quad \Pi=-\frac{\zeta}{\tau},
$$

where $\zeta$ and $\eta$ stand for bulk and shear viscosity, respectively.

\section{Bulk and shear viscosity and $\zeta / \eta$ scaling}

In order to obtain explicit formulas for viscosities in the GZ plasma one can use Eqs. (8)-(10) and consider small perturbations around equilibrium state. In this case one can write

$$
f \approx f_{\mathrm{GZ}}+\delta f+\cdots,
$$

which, when used together with the Boltzmann kinetic equation in the relaxation time approximation (RTA)

$$
\frac{\partial f\left(\tau, w, k_{\perp}\right)}{\partial \tau}=\frac{f_{\mathrm{GZ}}\left(\tau, w, k_{\perp}\right)-f\left(\tau, w, k_{\perp}\right)}{\tau_{\mathrm{rel}}(\tau)},
$$

after some algebra, leads to the following formulas

$$
\begin{gathered}
\zeta=\frac{g_{0} \gamma_{\mathrm{G}}^{5}}{3 \pi^{2}} \frac{\tau_{\mathrm{rel}}}{T} \int_{0}^{\infty} \mathrm{d} y\left[c_{s}^{2}-\frac{1}{3} \frac{y^{4}-1}{y^{4}+1}\right] f_{\mathrm{GZ}}\left(1+f_{\mathrm{GZ}}\right), \\
\eta=\frac{g_{0} \gamma_{\mathrm{G}}^{5}}{30 \pi^{2}} \frac{\tau_{\mathrm{rel}}}{T} \int_{0}^{\infty} \mathrm{d} y \frac{\left(y^{4}-1\right)^{2}}{y^{4}+1} f_{\mathrm{GZ}}\left(1+f_{\mathrm{GZ}}\right),
\end{gathered}
$$

for bulk and shear viscosity, respectively. Above $f_{\mathrm{GZ}}=\left\{\exp \left[\gamma_{\mathrm{G}} \sqrt{y^{2}+y^{-2}} / T\right]-1\right\}^{-1}$.

In the left panel of Fig. 1 we plot $\zeta$ and $\eta$ obtained from Eqs. (13)-(14) scaled by the equilibrium entropy density, $s$, and the relaxation time, $\tau_{\text {rel }}$, as functions of temperature. We observe that there is almost linear scaling of the scaled shear viscosity with temperature, similar to the ideal massless gas, $\eta / s=T / 5 \tau_{\text {rel }}$. We also observe a minor enhancement of bulk viscosity near the phase transition temperature $T_{c}=260 \mathrm{MeV}$. Another striking result is that, different from conventional quasiparticle approaches, at low temperatures bulk viscosity dominates over the shear viscosity.

Finally, in the right panel of Fig. 1 we show the $\zeta / \eta$ ratio scaled by the $1 / 3-c_{s}^{2}$. We observe that at large temperatures the scaling is, to a good approximation, linear. This can be seen more explicitly when one considers the large temperature expansion of the $\zeta / \eta$ ratio. In this case one obtains an exact linear scaling

$$
\frac{\zeta}{\eta}=\kappa_{\mathrm{GZ}}\left(\frac{1}{3}-c_{s}^{2}\right) \quad\left(T \gg \gamma_{\mathrm{G}}\right),
$$

where $\kappa_{\mathrm{GZ}}=5 / 2$. This is different from the perturbative treatments of the weakly-coupled plasmas, which predict a quadratic scaling [49-51]. Surprisingly, our result seems to be in line with the results obtained in strongly-coupled theories using gauge theory/gravity duality [52-54]. 

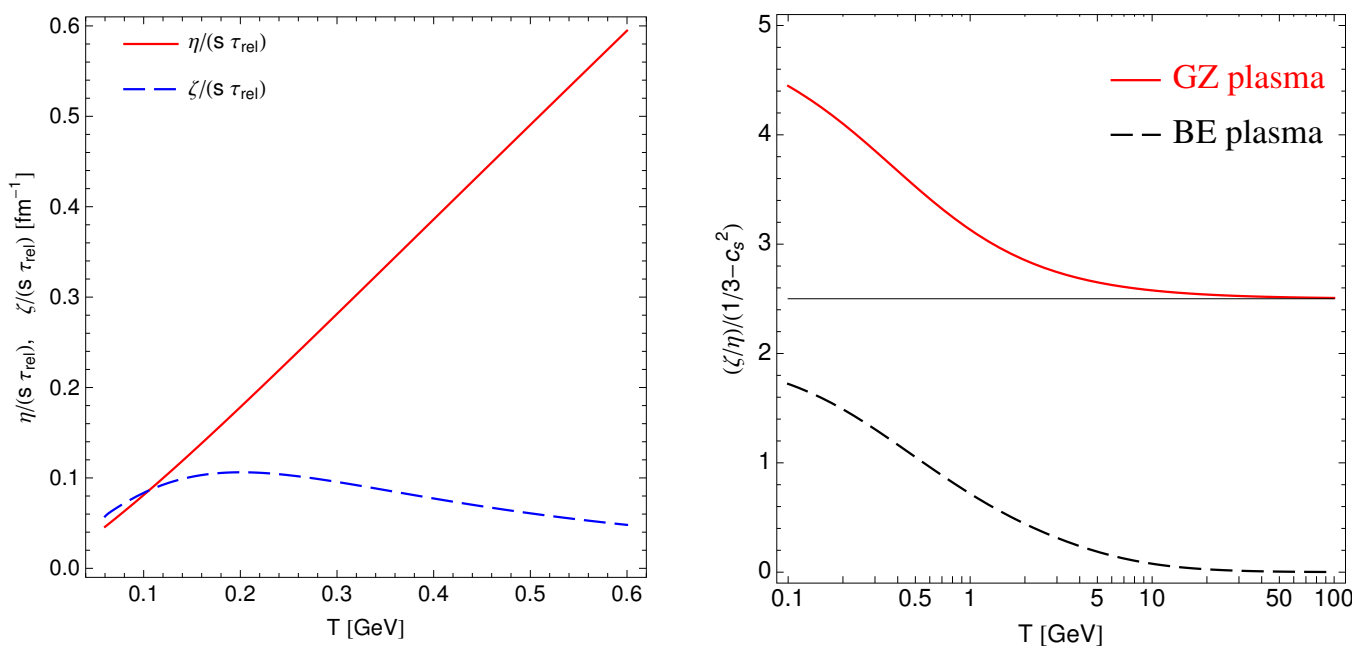

Figure 1: (Color online) Left: Analytic results on bulk (dashed blue line) and shear (solid red line) viscosity (see Eqs. (13) and (14), respectively) scaled by equilibrium entropy density and the relaxation time as functions of the temperature. Right: The bulk to shear viscosity ratio scaled by the difference $1 / 3-c_{s}^{2}$ for the Gribov-Zwanziger plasma and the Bose-Einstein plasma of massive particles with the mass $m=\sqrt{2} \gamma_{\mathrm{G}}$.

\section{Conclusions}

In this work we reviewed our recent results concerning the transport coefficients, namely shear and bulk viscosities, for a system composed of gluons resulting from the Gribov-Zwanziger quantization. The GZ procedure allows us to include the confining effects in the gluon system in a straightforwad way and, in consequence, to understand better the beviour of the SU(3) Yang-Mills plasma.

Using the fluid dynamical considerations in the small perturbation limit we found the exact analytic formulas for the transport coefficients. Based on the analysis of the obtained expressions we observe various properties of the system which are characteristic for the strongly-coupled systems. In particular, at large temperatures, we found the linear scaling of the $\zeta / \eta$ ratio with the difference $1 / 3-c_{s}^{2}$, which is similar to the one found in strongly-coupled non-conformal gauge theory plasma. Our findings stay in line with the well established understanding of the quark-gluon plasma as the strongly-coupled fluid with small viscosity.

\section{Acknowledgements}

Author would like to thank Wojciech Florkowski, Nan Su and Konrad Tywoniuk for the fruitful collaboration. This work was supported by the Polish National Science Center grant No. DEC-2012/07/D/ST2/02125.

\section{References}

[1] A. Muronga, Phys. Rev. Lett. 88, 062302 (2002), nucl-th/0104064

[2] U.W. Heinz, H. Song, A.K. Chaudhuri, Phys.Rev. C73, 034904 (2006), nucl-th/0510014 
[3] R. Baier, P. Romatschke, U.A. Wiedemann, Phys. Rev. C73, 064903 (2006), hep-ph/0602249

[4] K. Dusling, D. Teaney, Phys. Rev. C77, 034905 (2008), 0710. 5932

[5] M. Chojnacki, W. Florkowski, W. Broniowski, A. Kisiel, Phys. Rev. C78, 014905 (2008), 0712.0947

[6] M. Luzum, P. Romatschke, Phys. Rev. Lett. 103, 262302 (2009), 0901. 4588

[7] G.S. Denicol, T. Kodama, T. Koide, P. Mota, Phys. Rev. C80, 064901 (2009), 0903. 3595

[8] P. Bozek, Phys. Rev. C81, 034909 (2010), 0911. 2397

[9] A. El, Z. Xu, C. Greiner, Phys. Rev. C81, 041901 (2010), 0907.4500

[10] J. Peralta-Ramos, E. Calzetta, Phys.Rev. C82, 054905 (2010), 1003.1091

[11] B.H. Alver, C. Gombeaud, M. Luzum, J.Y. Ollitrault, Phys. Rev. C82, 034913 (2010), 1007.5469

[12] G. Denicol, T. Koide, D. Rischke, Phys.Rev.Lett. 105, 162501 (2010), 1004.5013

[13] B. Schenke, S. Jeon, C. Gale, Phys.Rev.Lett. 106, 042301 (2011), 1009. 3244

[14] P. Bozek, Phys.Rev. C85, 034901 (2012), 1110.6742

[15] H. Niemi, G.S. Denicol, P. Huovinen, E. Molnár, D.H. Rischke, Phys. Rev. C 86, 014909 (2012)

[16] P. Bozek, W. Broniowski, Phys. Rev. Lett. 109, 062301 (2012), 1204 . 3580

[17] I.A. Karpenko, Yu.M. Sinyukov, K. Werner, Phys. Rev. C87, 024914 (2013), 1204 . 5351

[18] J. Peralta-Ramos, E. Calzetta, Phys.Rev. D87, 034003 (2013), 1212.0824

[19] P. Bozek, A. Bzdak, V. Skokov, Phys. Lett. B728, 662 (2014), 1309.7358

[20] G. Denicol, S. Jeon, C. Gale, Phys.Rev. C90, 024912 (2014), 1403.0962

[21] F.G. Gardim, J. Noronha-Hostler, M. Luzum, F. Grassi, Phys. Rev. C91, 034902 (2015), 1411.2574

[22] R.S. Bhalerao, A. Jaiswal, S. Pal, Phys. Rev. C92, 014903 (2015), 1503.03862

[23] M. Martinez, M. Strickland, Nucl. Phys. A848, 183 (2010), 1007.0889

[24] W. Florkowski, R. Ryblewski, Phys. Rev. C83, 034907 (2011), 1007.0130

[25] L. Tinti, W. Florkowski, Phys. Rev. C89, 034907 (2014), 1312.6614

[26] D. Bazow, U.W. Heinz, M. Strickland, Phys. Rev. C90, 054910 (2014), 1311.6720

[27] D. Bazow, U.W. Heinz, M. Martinez, Phys.Rev. C91, 064903 (2015), 1503.07443

[28] M. Nopoush, M. Strickland, R. Ryblewski, D. Bazow, U. Heinz, M. Martinez, Phys. Rev. C92, 044912 (2015), 1506.05278

[29] L. Tinti (2015), 1506.07164

[30] A. Bazavov et al. (HotQCD), Phys. Rev. D90, 094503 (2014), 1407. 6387

[31] S. Borsanyi, Z. Fodor, C. Hoelbling, S.D. Katz, S. Krieg, K.K. Szabo, Phys. Lett. B730, 99 (2014), 1309. 5258

[32] J.O. Andersen, L.E. Leganger, M. Strickland, N. Su, JHEP 08, 053 (2011), 1103.2528

[33] S. Mogliacci, J.O. Andersen, M. Strickland, N. Su, A. Vuorinen, JHEP 12, 055 (2013), 1307.8098

[34] N. Haque, A. Bandyopadhyay, J.O. Andersen, M.G. Mustafa, M. Strickland, N. Su, JHEP 05, 027 (2014), 1402.6907

[35] H.B. Meyer, Eur. Phys. J. A47, 86 (2011), 1104 . 3708

[36] W. Florkowski, R. Ryblewski, N. Su, K. Tywoniuk (2015), 1504.03176

[37] W. Florkowski, R. Ryblewski, N. Su, K. Tywoniuk (2015), 1509.01242

[38] V.N. Gribov, Nucl. Phys. B139, 1 (1978)

[39] D. Zwanziger, Nucl. Phys. B323, 513 (1989) 
[40] D. Zwanziger, Phys. Rev. D76, 125014 (2007), hep-ph/0610021

[41] K. Fukushima, N. Su, Phys.Rev. D88, 076008 (2013), 1304.8004

[42] R. Alkofer, L. von Smekal, Phys. Rept. 353, 281 (2001), hep-ph/0007355

[43] M.N. Chernodub, V.I. Zakharov, Phys. Rev. Lett. 100, 222001 (2008), hep-ph/0703167

[44] G. Burgio, M. Quandt, H. Reinhardt, Phys. Rev. Lett. 102, 032002 (2009), 0807 . 3291

[45] A. Maas, Phys. Rept. 524, 203 (2013), 1106.3942

[46] N. Su, K. Tywoniuk, Phys. Rev. Lett. 114, 161601 (2015), 1409. 3203

[47] D.E. Kharzeev, E.M. Levin, Phys. Rev. Lett. 114, 242001 (2015), 1501.04622

[48] F.E. Canfora, D. Dudal, I.F. Justo, P. Pais, L. Rosa, D. Vercauteren, Eur. Phys. J. C75, 326 (2015), 1505.02287

[49] S. Weinberg, Astrophys. J. 168, 175 (1971)

[50] S. Jeon, L.G. Yaffe, Phys. Rev. D53, 5799 (1996), hep-ph/9512263

[51] W. Florkowski, A. Jaiswal, E. Maksymiuk, R. Ryblewski, M. Strickland, Phys. Rev. C91, 054907 (2015), 1503.03226

[52] P. Benincasa, A. Buchel, A.O. Starinets, Nucl. Phys. B733, 160 (2006), hep-th/0507026

[53] A. Buchel, Phys. Rev. D72, 106002 (2005), hep-th/0509083

[54] D. Li, S. He, M. Huang, JHEP 06, 046 (2015), 1411 . 5332 